\title{
Potência do ritmo alfa em pacientes com Transtorno do Espectro Autista e indivíduos controle durante realização de tarefas cognitivas
}

\author{
Alpha rhythm potency in patients with Autism Spectrum Disorder and control while \\ performing cognitive tasks
}

Potencia del ritmo alfa en pacientes con Trastorno del Espectro Autista y control mientras realizan tareas cognitivas

João Pedro Matos de Santana ${ }^{1 *}$, Lílian Santana Marcelino de Araújo², Morgana Rolemberg de Melo $^{3}$, Lousane Leonoura Alves Santos ${ }^{1}$, Milton Vieira Costa ${ }^{1}$, José Claudio da Silva ${ }^{1}$, Euclides Maurício Trindade Filho' ${ }^{1}$.

\section{RESUMO}

Objetivo: Comparar o mapeamento eletroencefalográfico entre voluntários com TEA e hígidos durante repouso e realização de tarefas cognitivas. Métodos: Foram estudados dois grupos: 9 voluntários com TEA e 20 hígidos entre 8 e 16 anos de ambos os sexos. Os participantes foram submetidos às fases de repouso, memorização, evocação de imagens, leitura e estimulações musicais 1 e 2. A partir disso, foram obtidos os potenciais alfa $(\alpha)$ nos quadrantes anterior esquerdo, anterior direito, posterior esquerdo e posterior direito, além de suas potências globais em cada etapa. Por fim, foi realizada a análise estatística através do programa Biostat 5.3, sendo aceito um nível de significância de $p \leq 0,05$. Resultados: Os pacientes com TEA demonstraram maiores valores de alfa nas etapas de repouso, evocação de figuras e estimulações musicais 1 e 2, o que envolveu tanto a análise segmentar como global. Estima-se que um prejuízo no funcionamento da atividade GABAérgica possa compor uma das bases do transtorno. Conclusão: $O$ estudo eletroencefalográfico demonstra o potencial de garantir possíveis biomarcadores úteis no diagnóstico do autismo, sendo necessários mais estudos com o intuito de definir melhor a relação entre análise espectral e fatores clínico-patológicos.

Palavras-chave: Transtorno do Espectro Autista, Neurociências, Desenvolvimento infantil.

\begin{abstract}
Objective: To compare the electroencephalographic mapping between volunteers with ASD and healthy subjects during rest and performing cognitive tasks. Methods: Two groups were studied: 9 volunteers with ASD and 20 healthy between 8 and 16 years old of both sexes. The participants were submitted to the phases of rest, memorization, image recall, reading and musical stimulation 1 and 2 . From this, the alpha $(\alpha)$ potentials were obtained in the anterior left, anterior right, posterior left and posterior right quadrants, in addition to their global powers in each stage. Finally, statistical analysis was performed using the Biostat 5.3 program, with a significance level of $p \leq 0.05$ being accepted. Results: Patients with ASD demonstrated higher alpha values in the rest, evocation of figures and musical stimulation 1 and 2 stages, both in terms of quadrant and overall value. It is estimated that an impairment in the functioning of GABAergic activity may make up one of the bases of the disorder. Conclusion: The electroencephalographic study demonstrates the potential to guarantee possible biomarkers useful in the diagnosis of autism, and further studies are needed in order to better define the relationship between spectral analysis and clinical-pathological factors.
\end{abstract}

Key words: Autistic Spectrum Disorder, Neuroscience, Child development.

\footnotetext{
${ }^{1}$ Universidade Estadual de Ciências da Saúde de Alagoas (UNCISAL), Maceió - AL.

*E-mail: jpedro 296@outlook.com

2 Universidade Tiradentes (UNIT), Aracaju - SE.

${ }^{3}$ Centro Universitário Cesmac, Maceió - AL.
}

Fundação de Amparo à Pesquisa do Estado de Alagoas (PIBIC/FAPEAL). 


\section{RESUMEN}

Objetivo: Comparar el mapeo electroencefalográfico entre voluntarios con TEA y sujetos sanos durante el descanso y la realización de tareas cognitivas. Métodos: Se estudiaron dos grupos: 9 voluntarios con TEA y 20 sanos entre 8 y 16 años de ambos sexos. Los participantes fueron sometidos a las fases de descanso, memorización, recuerdo de imágenes, lectura y estimulación musical 1 y 2 . A partir de esto, se obtuvieron los potenciales alfa $(\alpha)$ en los cuadrantes anterior izquierdo, anterior derecho, posterior izquierda y posterior derecha, además de sus poderes globales en cada etapa. Finalmente, el análisis estadístico se realizó mediante el programa Biostat 5.3, aceptando un nivel de significancia de $p \leq 0.05$. Resultados: Los pacientes con TEA demostraron mayores valores de alfa en el reposo, evocación de figuras y estimulación musical 1 y 2 etapas, que involucró análisis tanto segmentario como global. Se estima que un deterioro en el funcionamiento de la actividad GABAérgica puede constituir una de las bases del trastorno. Conclusión: EI estudio electroencefalográfico demuestra el potencial para garantizar posibles biomarcadores útiles en el diagnóstico del autismo, siendo necesarios más estudios para definir mejor la relación entre el análisis espectral y los factores clínico-patológicos.

Palabras clave: Trastorno del Espectro Autista, Neurociencia, Desarrollo infantil.

\section{INTRODUÇÃO}

O Transtorno do Espectro Autista (TEA) cursa com prejuízos mentais persistentes de modo a comprometer o desenvolvimento e a sociabilidade. Afeta $1 \%$ da população geral e apresenta maior frequência em meninos, estabelecendo uma relação de 4:1, que pode aumentar para 8:1 em casos nos quais não há déficit intelectual. Até os anos de 1980, as crianças acometidas pelo transtorno eram diagnosticadas frequentemente com esquizofrenia infantil, situação que foi ao longo do tempo atenuada tendo em vista a determinação mais precisa dos critérios diagnósticos em questão (KAPLAN H, et al., 2017).

Estima-se que o autismo conte com 2 pilares etiológicos: os fatores ambientais e genéticos. A influência ambiental cursa de maneira que infecções virais, como por citomegalovírus, herpes, rubéola e sarampo, podem favorecer o surgimento do transtorno. Por outro lado, a chance aumentada em 20 vezes de o segundo filho apresentar autismo quando o primeiro possui o transtorno e a concordância de 36 a $92 \%$ entre gêmeos monozigóticos são aspectos que ilustram a influência do aporte genético (CAVALHEIRA G, et al., 2004).

As características mais comuns associadas ao TEA são: o acometimento na comunicação verbal, de modo que inversões pronominais, ausência de prosódia e ecolalia ilustram essa situação; prejuízo à sociabilidade, que ocorre pela baixa capacidade de inferir estados mentais, ou seja, compreender emoções e sentimentos atrelados ao convívio social; e comportamentos estereotipados, o que está ligado ao prejuízo das funções executivas (KANDEL ER, 2014).

Além disso, irritabilidade, hipersensibilidade, desatenção e hiperatividade podem também estar presentes no transtorno. Associado a esse quadro clínico, há um conjunto de alterações encefálicas como déficits de desenvolvimento e funcionalidade no córtex pré-frontal, cerebelo, amígdala dentre outros locais (DONOVAN AP e BASSON M, 2017).

O tratamento atual ofertado consiste na atuação de uma equipe multidisciplinar que conta com médico, educador físico, fonoaudiólogo, terapeuta ocupacional dentre outros profissionais. Essa abordagem possui suma relevância para a melhoria das manifestações advindas do transtorno. Contudo, diante de sua complexidade característica, ainda não foi descoberta uma cura. Nesse sentido, a busca de ações mais eficazes a serem implementadas no tratamento perpassam por uma melhor compreensão da fisiopatologia envolvida (GARCIA SCM, et al., 2017).

Com esse objetivo, pode-se lançar mão de um importante método de monitoramento eletrofisiológico: a eletroencefalografia, que consiste no registro da atividade elétrica cerebral. Para tanto, ocorre a captação da onda cerebral, a qual representa a diferença de potencial elétrico entre dois eletrodos. A partir disso, torna-se possível fazer uma avaliação dos possíveis estados de excitabilidade neuronal, contribuindo, portanto, para uma análise de importância clínica (FEYISSA AM e TATUM WO, 2019).

Existem 5 tipos básicos de onda: alfa, beta, teta, delta e gama. As ondas delta apresentam frequência menor que $4 \mathrm{~Hz}$ e evidenciam-se nos estágios mais profundos do sono e em doença cerebral orgânica, estando 
ausente na vigília. Já as ondas teta cursam com frequência entre $4 \mathrm{~Hz}$ e $8 \mathrm{~Hz}$, sendo discretas na vigília e proeminentes em estados sonolentos, além de ocorrer não só em indivíduos normais como também em estados degenerativos (SILVA AAS e TRINDADE-FILHO EM, 2015).

As ondas alfa, alvo do presente estudo, incluem flutuações que variam na faixa de 8 a $13 \mathrm{~Hz}$ e está presente em quase todos os adultos em repouso. Ademais, é dominante na vigília com os olhos fechados, desaparecendo durante o sono profundo. As ondas beta possuem frequência maior que $13 \mathrm{~Hz}$ e surgem de modo assincrônico com baixa voltagem e elevada frequência quando o indivíduo volta sua atenção a alguma função específica. Por fim, as ondas gama, que variam entre 25 e $45 \mathrm{~Hz}$ e estão relacionados à execução de atividades cognitivas (SILVA AAS e TRINDADE-FILHO EM, 2015).

Apesar da vasta aplicabilidade do método em diversas situações, são necessários mais estudos acerca do papel do eletroencefalograma na identificação do autismo. Levando-se em consideração a possibilidade de elencar anormalidades corticais por meio do EEG, estima-se que o delineamento de alterações cerebrais realizado pelo exame pode contribuir com o melhor entendimento do quadro clínico em questão e, consequentemente, favorecer a compreensão do transtorno como um todo (GURAU O, et al., 2017).

Nesse sentido, a presente pesquisa possui como escopo realizar o mapeamento topográfico cerebral de pacientes com TEA e indivíduos controle pareado. Nessa esfera, objetiva-se comparar a atividade eletroencefalográfica em função do ritmo alfa entre os dois grupos durante o repouso e mediante a realização de tarefas cognitivas que contemplam leitura, música e memorização.

\section{MÉTODOS}

Consiste em um estudo quantitativo, observacional e transversal realizado em uma universidade pública de Alagoas. Foram incluídos no estudo voluntários diagnosticados com TEA (grupo teste) e participantes sem quaisquer distúrbios neuropsiquiátricos (grupo controle) de ambos os sexos, sendo excluídos indivíduos que apresentassem comportamento agressivo e/ou não colaborativo durante a realização do exame.

Nesse sentido, inicialmente foram recrutados por busca ativa um total de 31 voluntários de ambos os sexos (22 do sexo masculino e 9 do sexo feminino) na faixa etária entre 8 e 16 anos (Média: 11,1 / dp: 2,6), sendo 11 pacientes com TEA e 20 indivíduos hígidos (grupo controle). O espaço amostral foi baseado no estudo de Lazarev VV, et al. (2015) e houve a exclusão de 2 pacientes autistas que se encontravam agitados na ocasião do registro eletroencefalográfico, o que gerou o surgimento de artefatos em excesso de modo a prejudicar a interpretação do traçado.

Mediante a confirmação de interesse na presente pesquisa, os voluntários assinaram o Termo de Assentimento Livre e Esclarecido (TALE) e seus respectivos responsáveis assinaram o Termo de Consentimento Livre e Esclarecido (TCLE). A coleta ocorreu entre os meses de agosto de 2019 e janeiro de 2020 no Laboratório de Neurociências da supracitada instituição entre 14h e 18h posteriormente à aprovação do projeto pelo Comitê de Ética em Pesquisa (CEP), sob número CAAE: 02042918.1.0000.5011 e parecer de n 3.353.465. O trabalho atendeu às exigências da Resolução CNS n 466/2012.

Inicialmente, os indivíduos preencheram um formulário com informações sociodemográficas (idade e sexo). Em seguida, os participantes sentaram em cadeira confortável e foi iniciada a colocação dos eletrodos do eletroencefalograma. O aparelho utilizado foi da marca EMSA, modelo 2031, de 20 canais, processador de 12 bits e frequência amostral de 256 pontos. A distribuição dos eletrodos no escalpo obedeceu ao sistema internacional 10-20, com 22 eletrodos (Fp1/Fp2, F7/F8, T3/T4, T5/T6, F3/ F4, C3/C4, P3/P4, O1/O2, FZ, CZ, $\mathrm{PZ}$ e OZ, A1/ A2).

A atividade elétrica foi catalogada durante seis etapas. A primeira foi a fase de repouso, momento no qual o voluntário permanecia de olhos fechados durante 1 minuto para realização do registro basal. Em seguida, iniciou-se a tarefa de memorização de imagens, na qual foram apresentadas 5 figuras ilustrativas (bola, banana, carro, cadeira e árvore) durante 30 segundos. Por conseguinte, após um novo período de 30 segundos de olhos fechados, foi executada a terceira fase, na qual o participante deveria evocar as imagens demonstradas na etapa anterior. 
Já a quarta etapa consistia na leitura de uma frase simples ("A mãe chegou em casa e a criança estava brincando"). Por fim, durante as duas últimas fases o voluntário era exposto a trechos de músicas clássicas, sendo a quinta fase correspondente à canção Spring Waltz (Paul de Seneville) e a sexta referente à $5^{\mathrm{a}}$ sinfonia de Ludwig van Beethoven.

Após a finalização do exame, o ritmo alfa ( $\alpha$ ) foi analisado de maneira comparativa entre os autistas e controle pareado nos quadrantes Anterior Esquerdo (QAE), Anterior Direito (QAD), Posterior Esquerdo (QPE) e Posterior Direito (QPD). Além disso, também foi avaliada a potência global, que consiste na média aritmética das potências alfa de todo o córtex cerebral durante determinada etapa e em determinado grupo (autistas ou controle).

Foram aplicadas técnicas de estatísticas descritivas incluindo tabelas. Na estatística inferencial a comparação entre os dois grupos foi realizada através do teste T'Student caso os pressupostos de normalidade e homocedasticidade sejam atendidos. Para amostras independentes, foi utilizado o teste não paramétrico de Mann-Witney. Por fim, foi aceito como significante um valor de $p \leq 0,05$.

\section{RESULTADOS}

O estudo se concentrou na análise das ondas alfa em voluntários com TEA (grupo teste) e indivíduos controle de maneira comparativa. Aplicando-se os critérios de inclusão e exclusão, a presente pesquisa contou com 20 voluntários hígidos e 9 autistas, sendo a maioria do sexo masculino (69\%). Não houve participantes do sexo feminino no grupo teste, o que pode ilustrar a clara desproporção entre homens e mulheres no transtorno.

No que tange à análise segmentar do grupo controle, a leitura representou a tarefa com maior potência alfa nos quadrantes anterior esquerdo (10), anterior direito (9.8) e posterior esquerdo (9.9). Ademais, essa mesma fase igualou-se à etapa de evocação de imagens no quadrante posterior direito (9.8). Quanto ao grupo teste, as músicas clássicas 1 e 2 em conjunto perfizeram os maiores valores no quadrante anterior esquerdo (10.2), o repouso no quadrante anterior direito (10.4), a música 2 no quadrante posterior esquerdo (10.1) e a leitura juntamente à música 2 no quadrante posterior direito (9.9). Todos os valores de potência alfa por cada segmento cortical encontram-se na Tabela 1.

Tabela 1 - Potência do ritmo alfa durante repouso e tarefas cognitivas de acordo com os quadrantes corticais.

\begin{tabular}{|c|c|c|c|c|c|c|c|c|}
\hline \multirow{2}{*}{ Etapas } & \multicolumn{4}{|c|}{ Controle } & \multicolumn{4}{|c|}{ TEA } \\
\hline & QAE & QAD & QPE & QPD & QAE & QAD & QPE & QPD \\
\hline \multirow{2}{*}{ Repouso } & 9.7 & $9.7^{*}$ & 9.3 & 9.6 & 10.1 & $10.4^{\star}$ & 9.3 & 9.7 \\
\hline & \pm 0.7 & \pm 0.7 & \pm 0.9 & \pm 0.7 & \pm 1.1 & \pm 0.7 & \pm 0.8 & \pm 0.8 \\
\hline \multirow{2}{*}{ Memorização } & 9.6 & 9.7 & 9.4 & 9.7 & 10.1 & 9.8 & 9.4 & 9.6 \\
\hline & \pm 0.7 & \pm 0.7 & \pm 0.8 & \pm 0.7 & \pm 1.1 & \pm 0.7 & \pm 0.9 & \pm 0.7 \\
\hline \multirow{2}{*}{ Evocação } & 9.8 & $9.6^{\star *}$ & 9.6 & 9.8 & 9.9 & $10.3^{\star \star}$ & 9.8 & 9.8 \\
\hline & \pm 1.0 & \pm 0.8 & \pm 0.7 & \pm 0.4 & \pm 0.8 & \pm 1.0 & \pm 0.8 & \pm 0.8 \\
\hline \multirow{2}{*}{ Leitura } & 10.0 & 9.8 & 9.9 & 9.8 & 9.9 & 10.1 & 9.9 & 9.9 \\
\hline & \pm 0.7 & \pm 0.8 & \pm 0.9 & \pm 0.7 & \pm 0.6 & \pm 1.3 & \pm 0.7 & \pm 0.5 \\
\hline \multirow{2}{*}{ Música 1} & $9.4^{\star \star \star}$ & 9.7 & 9.4 & 9.4 & $10.2^{\star \star \star}$ & 10.1 & 9.5 & 9.7 \\
\hline & \pm 0.8 & \pm 0.7 & \pm 1.0 & \pm 0.8 & \pm 1.1 & \pm 1.0 & \pm 0.9 & \pm 0.9 \\
\hline \multirow{2}{*}{ Música 2} & $9.2^{\star \star \star \star}$ & 9.5 & $8.9^{\star \star \star \star \star \star}$ & $9.4^{\star \star \star \star \star \star}$ & $10.2^{\star \star \star \star}$ & 9.9 & $10.1^{\star \star \star \star \star \star}$ & $9.9^{\star \star \star \star \star \star \star ~}$ \\
\hline & \pm 0.7 & \pm 0.7 & \pm 0.8 & \pm 0.7 & \pm 1.0 & \pm 1.1 & \pm 0.4 & \pm 0.7 \\
\hline
\end{tabular}


Acerca da análise global, o grupo controle contou com potencial mais expressivo na fase de leitura (9.83), seguida das etapas de evocação (9.67), memorização (9.58), repouso (9.53), estimulação musical 1 (9.45) e estimulação musical 2 (9.25). Por sua vez, o maior valor encontrado no grupo de voluntários autistas foi observado durante a música 2 (10.02), que foi seguida pelas fases de evocação (9.97), leitura (9.97), repouso (9.87), estimulação musical 1 (9.86) e, por fim, memorização (9.72). A comparação das potências globais encontra-se na Tabela 2.

Tabela 2 - Potência global do ritmo alfa nos grupos teste e controle durante repouso e tarefas cognitivas.

\begin{tabular}{cccc}
\hline Etapas & Controle & TEA & Valor de $\mathbf{p}$ \\
\hline Repouso & $9.53 \pm 0.8$ & $9.87 \pm 0.9$ & 0.061 \\
Memorização & $9.58 \pm 0.7$ & $9.72 \pm 0.9$ & 0.49 \\
Evocação & $9.67 \pm 0.7$ & $9.97 \pm 0.9$ & $\mathbf{0 . 0 0 8}$ \\
Leitura & $9.83 \pm 0.8$ & $9.97 \pm 0.8$ & 0.47 \\
Música 1 & $9.45 \pm 0.8$ & $9.86 \pm 1.0$ & $\mathbf{0 . 0 1 3}$ \\
Música 2 & $9.25 \pm 1.0$ & $10.02 \pm 0.8$ & $<\mathbf{0 . 0 0 0 1}$ \\
\hline
\end{tabular}

Fonte: Santana JPM, et al., 2021.

Foi identificada diferença significativa em sua distribuição em quatro das seis etapas realizadas, a saber: repouso, evocação de imagens, estimulação musical 1 e estimulação musical 2. As ondas alfa demonstraram maiores potenciais em pacientes com TEA ao longo de todas as 4 fases cuja divergência entre os grupos foi estatisticamente significante.

Dessa maneira, houve importante divergência no QAD durante a fase de repouso $(p=0,03)$. Quanto à etapa de evocação de figuras referente ao grupo controle, dezessete voluntários $(85 \%)$ acertaram cinco imagens e três (15\%) acertaram três imagens. Por sua vez, nessa mesma fase, quatro autistas $(44,4 \%)$ conseguiram evocar cinco figuras, três $(33,3 \%)$ relembraram quatro imagens, um $(11,1 \%)$ acertou três figuras e um $(11,1 \%)$ não conseguiu evocar nenhuma imagem.

Notou-se uma média de acerto no grupo controle de 4,7 imagens, valor acima do encontrado no grupo de autistas (3,8 figuras), apesar de não demonstrar relevância estatística quando comparados os valores brutos entre si $(p=0,10)$. Entretanto, durante a análise dessa mesma etapa, o potencial alfa segmentar foi significantemente maior no QAD do grupo teste $(p=0,011)$.

Da mesma forma, a música 1 foi capaz de gerar valores significantes no QAE $(p=0,019)$, enquanto a música 2 demonstrou alta significância no QPD $(p=0,042)$ e em todo o hemisfério esquerdo, envolvendo $o$ QAE $(p=0,007)$ e, especialmente, o QPE $(p=0,0008)$. Percebeu-se, ainda, que a potência de alfa global foi significantemente maior nos autistas durante as etapas de evocação de figuras $(p=0.008)$ e estimulação com a música clássica $1(p=0,013)$ e música clássica $2(p<0.0001)$.

\section{DISCUSSÃO}

Sabe-se que as ondas alfa variam de acordo com situações de relaxamento, processos cognitivos e emocionais. Nesse sentido, tendem a ser melhor visualizadas durante o repouso (MERRIT HH, 2011), além de apresentarem maiores potenciais na região frontal de indivíduos com maior capacidade de regulação emocional (DISTEFANO C, et al., 2019). Por outro lado, estados cognitivos como atenção, orientação e memória atenuam esse ritmo, o qual também varia de acordo com a idade de maneira que é mais proeminente em adultos do que na faixa etária infantil. (EDGAR JC, et al., 2015). Ademais, sugere-se que o ritmo alfa possui relação com atividades corticais e subcorticais, sendo possivelmente geradas nas alças tálamo-córticotalâmicas (KAY BP, et al., 2012).

Na presente pesquisa, os pacientes com TEA demonstraram maior valor de alfa durante o repouso no QAD, o que se aproxima de outros estudos que avaliaram essa mesma etapa. Nesse contexto, Cornew L, et al. (2012) encontraram maior atividade desse ritmo nas regiões temporal e parietal de autistas, além de associação direta entre esses achados e a gravidade dos sintomas. Por sua vez, Edgar JC, et al. (2015) em seu estudo com 41 autistas e 47 indivíduos controles (faixa etária: 6-14 anos) averiguaram aumento da atividade alfa do hemisfério esquerdo de pacientes com TEA, sendo associado a maior prejuízo social. 
Outras pesquisas perceberam a presença de um padrão de ondas em "U" no EEG de autistas durante o repouso. Nesse cenário, as ondas de baixa frequência (teta e delta) e de alta frequência (gama e beta) apresentariam aumento nos voluntários com TEA, enquanto ritmos de média frequência (alfa) contariam com redução nesse mesmo grupo. Estima-se que tal fato seja justificado por um funcionamento anormal da atividade GABAérgica em sua atuação inibitória, influenciando na plasticidade cerebral e no neurodesenvolvimento. Nota-se, nessa esfera, que o presente estudo constitui um contraponto ao supracitado tendo em vista que foi observada uma elevação do ritmo alfa no grupo teste quando comparado ao controle (WANG J, et al., 2013).

Durante a tarefa de evocação de figuras, o grupo de autistas demonstrou maior atividade alfa no quadrante anterior direito e na potência global. Levando-se em consideração que esse ritmo costuma estar reduzido durante a execução de tarefas, a maior atividade alfa averiguada no presente estudo constitui outro contraponto ao encontrado na literatura vigente.

Não foram observadas diferenças significantes durante as etapas de memorização e de leitura. Apesar disso, sabe-se que a compreensão linguística constitui um grande déficit nos pacientes com o transtorno, sendo de suma importância o papel da intervenção precoce, e que a memória de trabalho se encontra potencialmente reduzida em indivíduos com TEA (GRIMM RP, et al., 2018; LARRAIN-VALENZUELA J, et al., 2017).

As maiores divergências entre os grupos foram encontradas durante as fases de estimulação com músicas clássicas. Nessa esfera, a música 1 acarretou em maiores valores no grupo de autistas na potência global e no QAE, enquanto a música 2 ocasionou maior atividade alfa no QAE, QPE, QPD e na potência global. Em linha gerais, o processamento musical consiste em uma das habilidades mais complexas dentro do estudo da cognição humana. Tal função envolve diversas estruturas encefálicas, como o giro temporal superior, giro supramarginal, ínsula, córtex orbitofrontal, hipocampo, amígdala, cerebelo dentre outros componentes (CARIA A, et al., 2011). No contexto da análise espectral, sabe-se que a atividade alfa pode estar reduzida durante a estimulação musical.

Em estudo que avaliou a influência da $5^{\text {a }}$ sinfonia de Ludwig van Beethoven no traçado eletroencefalográfico de voluntários hígidos, notou-se relação direta entre excitabilidade emocional e supressão de alfa nas regiões frontal direita e parietotemporal esquerda (MIKUTTA C, et al., 2012). Em contraponto a esse estudo, Schaefer RS, et al. (2011) averiguaram que a atividade alfa durante a tarefa musical se eleva em indivíduos de neurodesenvolvimento típico e varia de acordo com o tipo e complexidade desse estímulo.

Quanto aos pacientes com TEA, encontra-se bastante consolidada na literatura a dificuldade dos indivíduos supracitados em compreender fatores emocionais atrelados às relações humanas, o que contempla as expressões faciais, gestos, expressões idiomáticas dentre outros componentes. Entretanto, a associação entre o autismo e o processamento musical ainda encontra importantes divergências (CARIA A, et al., 2011).

Em estudo realizado com 22 voluntários (8 autistas e 14 hígidos) que foram expostos a músicas tristes e alegres, não foram observadas diferenças importantes na deteç̧ão do componente emocional entre os dois grupos durante a tarefa. Dessa maneira, estima-se que a capacidade de processamento das emoções na esfera da música aparenta estar preservada, destoando do observado durante as relações interpessoais (CARIA A, et al., 2011).

Em outro estudo realizado com 40 indivíduos entre 17 e 34 anos, sendo 20 autistas e 20 voluntários do grupo controle, observou-se melhor percepção auditiva no grupo dos participantes com TEA (REMINGTON A e FAIRNIE J, 2017). Por outro lado, Sota S, et al. (2018) observaram em seu estudo que a habilidade musical em grande parte dos autistas encontra-se prejudicada, expressando relação direta entre pior memória de trabalho, desatenção e hiperatividade com um processamento auditivo deficitário.

Desse modo, percebe-se que ainda se conhece pouco acerca de como o estímulo afetivo não social, como é o caso da música, é processado nesses pacientes, assim como permanece uma incógnita a relação genérica entre padrão do ritmo alfa e autismo (CARIA A, et al., 2011). 
Estima-se, atualmente, que a musicoterapia alcança importantes benefícios para pacientes com TEA tendo em vista seu potencial de promover a neuroplasticidade por meio de uma reorganização estrutural e funcional das substâncias branca e cinzenta (BEDETTI C, et al., 2018). Dessa maneira, pode-se projetar como um importante modulador das emoções, efetuando melhora clínica superior a simples exposição à música (MIKUTTA C, et al., 2012; RABEYRON T, et al, 2020).

Nesse contexto, um ensaio clínico randomizado canadense corrobora com o fato supracitado. Nesse estudo, 51 voluntários autistas entre 6 e 12 anos foram recrutados, dos quais 26 foram submetidos à musicoterapia e 25 não receberam qualquer tipo de intervenção. Após um período de 8 a 12 semanas, notouse melhora nas habilidades sociais e na conectividade frontotemporal daqueles que participaram da terapia musical quando comparados ao grupo controle (SHARDA M, et al., 2018).

Por fim, é possível notar que ainda existe uma elevada divergência entre os estudos no que tange à análise espectral comparativa entre autistas e indivíduos saudáveis. Tal cenário pode ser atribuído a uma série de aspectos, que inclui principalmente a variedade de desenhos de estudo, faixa etária contemplada, presença ou não de deficiência cognitiva, comportamento agitado dos participantes dentre outros aspectos que podem dificultar a reprodutibilidade dos achados.

\section{CONCLUSÃO}

Foi evidenciado na presente pesquisa que a análise espectral demonstra potencial de garantir possíveis biomarcadores úteis na compreensão do autismo. Nesse contexto, puderam ser visualizadas importantes divergências no ritmo alfa quando comparados os voluntários com TEA e o grupo controle. Em contrapartida, a intersecção entre o registro eletroencefalográfico e as características clínicas do transtorno ainda constitui uma incógnita, o que pode ser decorrente das diferenças metodológicas dos estudos e da heterogeneidade neurológica típica desse grupo.

\section{REFERÊNCIAS}

1. BEDETTI C, et al. Mozart's music and multidrug-resistant epilepsy: a potential EEG index of therapeutic effectiveness. Psychiatr Danub, 2018; 30: 567- 571.

2. CARIA $A$, et al. Functional and dysfunctional brain circuits underlying emotional processing of music in autism spectrum disorders. Cerebral Cortex, 2011; 21 (12): 2838-2849.

3. CARVALHEIRA G, et al. Genética do autismo. Rev. Bras. Psiquiatr., 2004; 26 (4): 270-272.

4. CORNEW L, et al. Resting-state oscillatory activity in autism spectrum disorders. Journal of autism and developmental disorders, 2012; 42 (9): 1884-1894.

5. DISTEFANO C, et al. EEG data collection in children with ASD: The role of state in data quality and spectral power. Research in autism spectrum disorders, 2019; 57: 132-144.

6. DONOVAN APA, BASSON MA. The neuroanatomy of autism-a developmental perspective. Journal of anatomy, 2017; 230(1): 4-15.

7. EDGAR JC, et al. Resting-state alpha in autism spectrum disorder and alpha associations with thalamic volume. Journal of autism and developmental disorders, 2015; 45 (3): 795-804.

8. FEYISSA AM, TATUM WO. Adult EEG. Handbook of Clinical Neurology, 2019; 103-124.

9. GARCI SCM, et al. Autismo infantil: acolhimento e tratamento pelo sistema único de saúde. Revista Científica da Faculdade Fluminense, 2017; 2(1).

10. GRIMM RP, et al. Comparing growth in linguistic comprehension and reading comprehension in school-aged children with autism versus typically developing children. Autism Research, 2018; 11 (4): 624-635.

11. GURAU O, et al. How Useful Is Electroencephalography in the Diagnosis of Autism Spectrum Disorders and the Delineation of Subtypes: A Systematic Review. Frontiers in Psychiatry, 2017; 8: 121, 2017.

12. GUYTON AC, HALL JE. Tratado de fisiologia médica. 12. ed. Rio de Janeiro: Elsevier, 2011.

13. KANDEL ER. Princípios de neurociências. 5. ed. Porto Alegre: AMGH, 2014.

14. KAPLAN H, et al. Compêndio de Psiquiatria. 11 $1^{\text {a }}$ ed. Porto Alegre: Artmed, 2017.

15. KAY BP, et al. Moderating effects of music on resting state networks. Brain research, $2012 ; 1447: 53-64,2012$.

16. LARRAIN-VALENZUELA J, et al. Theta and alpha oscillation impairments in autistic spectrum disorder reflect working memory deficit. Scientific reports, $2017 ; 7$ (1): 1-11.

17. LAZAREV VV, et al. Reduced Interhemispheric Connectivity in Childhood Autism Detected by Electroencephalographic Photic Driving Coherence. J Autism Dev Disord, 2015.

18. MCFADDEN KL, et al. Abnormalities in gamma-band responses to language stimuli in first-degree relatives of children with autism spectrum disorder: an MEG study. BMc psychiatry, 2012; 12 (1): 1-18. 
19. MERRITT HH. Tratado de neurologia. 12. ed. Rio de Janeiro: Guanabara Koogan, 2011.

20. MIKUTTA C, et al. Emotions, arousal, and frontal alpha rhythm asymmetry during Beethoven's 5th symphony. Brain topography, 2012; 25 (4): 423-430.

21. RABEYRON T, et al. A randomized controlled trial of 25 sessions comparing music therapy and music listening for children with autism spectrum disorder. Psychiatry Research, 2020; 293: 13377.

22. REMINGTON A, FAIRNIE J. A sound advantage: Increased auditory capacity in autism. Cognition, 2017; 166: 459465.

23. SCHAEFER RS, et al. Music perception and imagery in EEG: Alpha band effects of task and stimulus. International Journal of Psychophysiology, 2011; 82 (3): 254-259.

24. SHARDA M, et al. Music improves social communication and auditory-motor connectivity in children with autism. Translational psychiatry, 2018; 8(1): 1-13.

25. SILVA AAS, TRINDADE-FILHO EM. Diferenças no processamento cerebral, através do ritmo gama, durante 0 pensamento divergente. Rev Neurocienc, 2015; 4:.589-594.

26. SOTA S, et al. Musical disability in children with autism spectrum disorder. Psychiatry research, 2018; 267: 354-359.

27. WANG J, et al. Resting state EEG abnormalities in autism spectrum disorders. Journal of neurodevelopmental disorders, 2013; 5(1):1-14. 